\title{
Goserelin Acetate
}

National Cancer Institute

\section{Source}

National Cancer Institute. Goserelin Acetate. NCI Thesaurus. Code C1417.

The acetate salt of a synthetic decapeptide analog of luteinizing hormone-releasing hormone (LHRH). Continuous, prolonged administration of goserelin in males results in inhibition of pituitary gonadotropin secretion, leading to a significant decline in testosterone production; in females, prolonged administration results in a decrease in estradiol production. ( $\mathrm{NCl04)}$ 